\title{
Commissioning of the Wendelstein 7-X In Vessel Control Coils
}

\author{
Frank Füllenbach, K. Risse, T. Rummel, P. v. Eeten, A. Carls, O. Volzke, M. Haas, H.-S. Bosch \\ and the W7-X Team
}

\begin{abstract}
The magnet system of the stellarator fusion device Wendelstein 7-X (W7-X) is composed of three different groups of coil systems. The main magnetic field is created by a superconducting magnet system that is accompanied by two sets of normal conducting coil groups, the trim coils positioned outside of the cryostat and the control coils inside the plasma vessel. The control coil system consists of ten 3D shaped coils, power supplies, cooling systems, high current feeds and an autonomous remote control system. The coils are situated behind the baffle plates of the ten divertor units. The magnetic field created by the control coils system allow for the correction of error fields, to influence the islands at the plasma boundary and for the sweeping of the separatrix, e.g. the point of the largest power position, across the divertor. At the end of 2015 the installation of the control coil system was completed and the integral commissioning took place in parallel to the ongoing completion of W7-X. For the first time the control coils and their power supply were operated in conjunction with all auxiliary systems like the power distribution system, the high current feeds, the cooling system and the safety control system. This paper describes the results obtained and experiences made during the integral commissioning of the control coil system, including the baking process in preparation for the first experimental campaign of $\mathrm{W} 7-\mathrm{X}$.
\end{abstract}

Index Electro magnets, Fusion power generation, Power electronic, Converters

\section{INTRODUCTION}

$\mathrm{T}$ $\mathrm{HE} \mathrm{W} 7-\mathrm{X}$ is a magnetic confinement stellarator fusion machine based upon a superconducting magnet system with a fivefold symmetry. Beside the plasma physics challenges of stellarator plasmas in steady state operation, the W7-X shall demonstrate the feasibility of an advanced reactor relevant magnet system within the optimized stellarator line [1].

In addition to the superconducting magnet system the W7-X is equipped with two sets of normal conducting coils, the Trim Coils and the Control Coils (CC) whereas the latter are the

Paper submitted 27.06.2017 This work has been carried out within the framework of the EUROfusion Consortium and has received funding from the Euratom research and training programme 2014-2018 under grant agreement No 633053. The views and opinions expressed herein do not necessarily reflect those of the European Commission.

All the authors are is with the Max Planck Institut für Plasmaphysik Wendelsteinstraße 1 17491 Greifwald Germany (e-mail: frank.fuellenbach@ipp.mpg.de) scope of this paper.

The CC is designed to add a dc and an ac component to the main magnetic field in order to:

- control and modify the size and the position of the islands at the boundary

- correct symmetry-breaking error fields and eliminate the corresponding islands

- influence the ergodicity of field lines for dispersal of magnetic surfaces at the boundary

- sweep the hot spots on the target plates avoiding excessive temperatures

The magnetic compensating capacity of a $\mathrm{CC}$ is shown in table 1 .

TABLE I

COMPENSATING CAPACITY OF ONE CONTROL COIL

\begin{tabular}{lcccc}
\hline \hline \multirow{2}{*}{ Control Coil } & $\mathrm{B}_{11} / 3 \mathrm{~T}$ & $\mathrm{~B}_{22} / 3 \mathrm{~T}$ & $\mathrm{~B}_{33} / 3 \mathrm{~T}$ & $\mathrm{~B}_{44} / 3 \mathrm{~T}$ \\
& $10^{-4}$ & $10^{-4}$ & $10^{-4}$ & $10^{-4}$ \\
\hline $20 \mathrm{kA}$ & 1.07 & 2.67 & 3.47 & 2.93 \\
\hline \hline
\end{tabular}

The compensating capacity is shown for the first four Fourier field components normalized at $3 \mathrm{~T}$ for the full $\mathrm{CC}$ current times no. of turns.

There is one $\mathrm{CC}$ installed behind the baffles plates of each of the ten divertor modules inside the plasma vessel. Fig. 1 shows the $\mathrm{CC}$ in lower left corner.

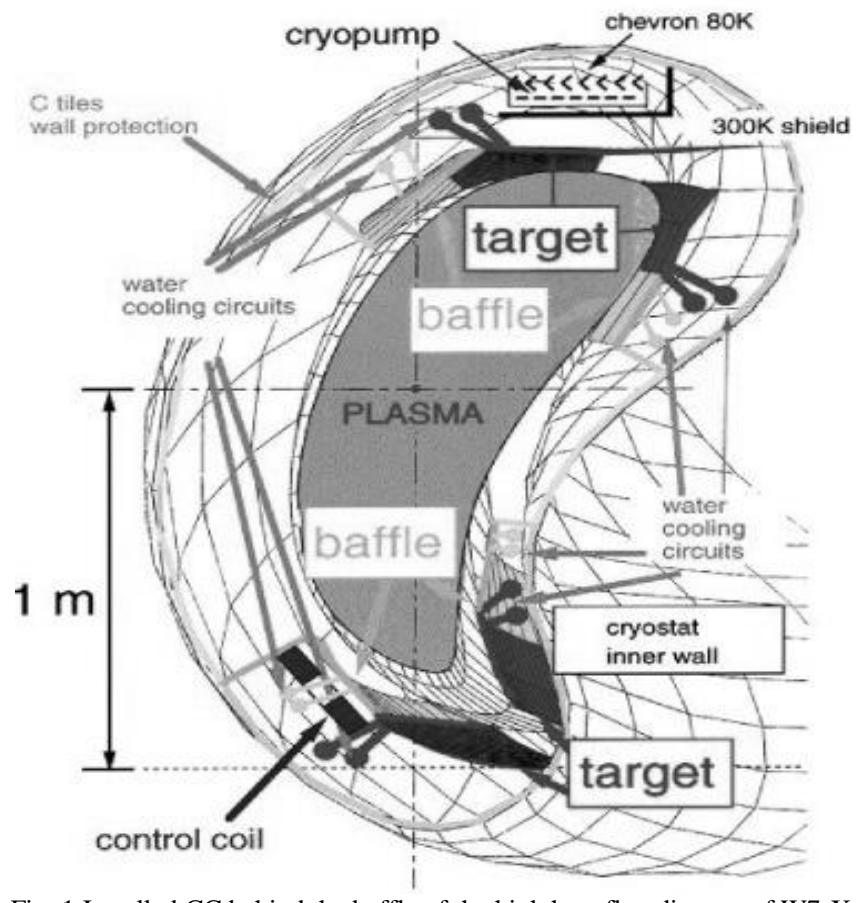

Fig. 1 Installed CC behind the baffle of the high heat flux divertor of W7-X 


\section{THE CONTROL COIL SySteM}

The CC system is comprised of the ten identical coils with its feed through inside a plug-in, power lines to the power supply with its cooling and control system, cooling for the coil and an inter space vacuum for the connection between feed through and coil housing.

\section{A. The Coil}

The $\mathrm{CC}$ is a normal conducting copper coil build upon a double pancake with 8 turnss. The conductor is a square hollow profile with a cross section of $16 \mathrm{~mm} \times 16 \mathrm{~mm}$. The central current path is three dimensional and follows the plasma vessel geometry. The coil winding is embedded in a coil housing of $3 \mathrm{~mm}$ stainless steel which is attached to the plasma vessel with three bearings. The central bearing is fixed whereas the both outside bearings can take on lateral thermal expansion of the coil. In Figure 2 a CAD view of the $\mathrm{CC}$ with the plug-in is shown [2].

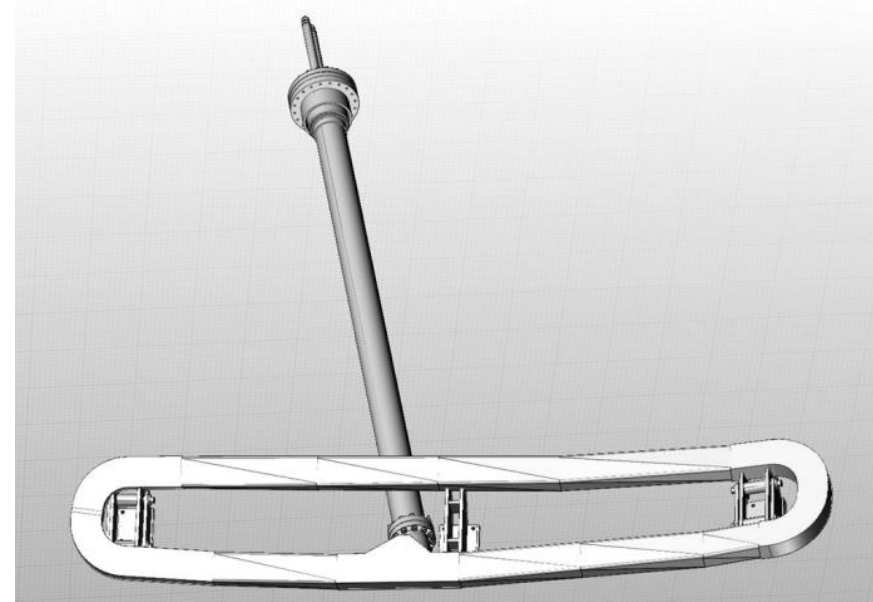

Fig. 2 Control Coil in its housing with the attached bearings and the mounted feed through in its plug-in.

The main parameters of the coil with its housing including the feed through in the plug-in are shown in table 2.

TABLE 2

MAIN PARAMETERS OF ONE CONTROL COIL

\begin{tabular}{lc}
\hline \hline \multicolumn{1}{c}{ Designation } & Value \\
\hline mass (winding/total) & $68 \mathrm{~kg} / 150 \mathrm{~kg}$ \\
inductance & $160 \mu \mathrm{H}$ \\
resistance @ RT & $3.95 \mathrm{~m} \Omega$ \\
power losses & $26 \mathrm{~kW}$ \\
max. temperature operation & $600^{\circ} \mathrm{C}$ \\
max. temperature baking & $160^{\circ} \mathrm{C}$ \\
\hline \hline
\end{tabular}

\section{B. The Feed Through}

The feed through connects the coil with the supply systems. It is situated inside a plug-in that seals the plasma vessel vacuum against the ambient and delivers cooling water and electrical energy by means of two hollow conductors. The connection between the plug-in and the feed through and coil housing is shown in Fig. 3 [2].

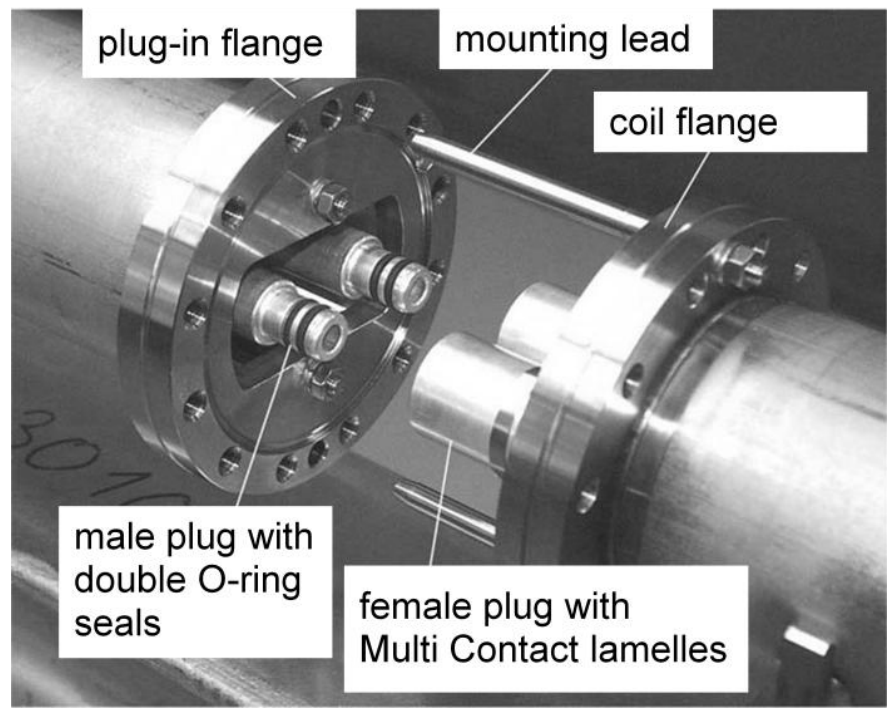

Fig. 3 Connection of the plug-in and feed through on the left side and winding with coil housing on the right side.

The connection to the current cables is done by a clamped copper block which is also equipped with contact lamellas for secure current conduction.

\section{The Power Supply}

To provide the bidirectional dc current and the superimposed ac current for sweeping, the power supply is designed as a four quadrant current supply based upon a water cooled Mosfet converter in H-bridge configuration. Each coil is supplied by one individual power supply unit (PSU). Fig. 4 shows the circuit scheme of one PSU.

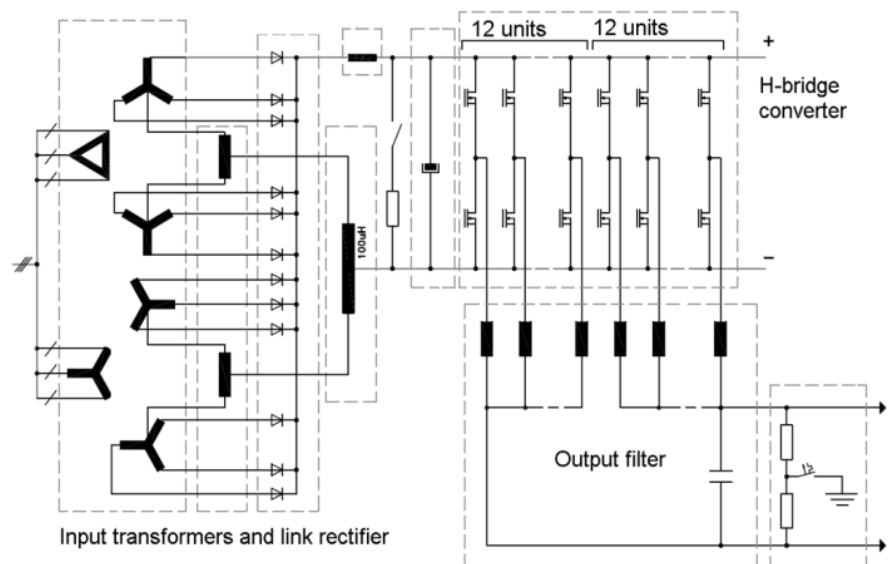

Fig. 4 Circuit scheme of one PSU the two input transformers, the DC link rectifier and interphase coils followed by the H-bridge current converter with a filter and grounding unit.

The whole power supply system contains its own cooling water plant for deionized water and a control system for autonomous und sub ordinated control. Energy distribution to each coil is done by means of four high cross-section cables in parallel minimizing the voltage drop. The main electric parameters of one PSU are shown in Table 3 [3]. 
TABLE 3

ELECTRIC PARAMETERS FOR ONE PSU

\begin{tabular}{lc}
\hline \hline \multicolumn{1}{c}{ Designation } & Value \\
\hline dc component of the current & $-2500 \mathrm{~A} \ldots+2500 \mathrm{~A}$ \\
ac component of the current & $0 \ldots 625 \mathrm{~A}_{\mathrm{P}}$ \\
Max eff. current & $2539 \mathrm{~A}$ \\
Frequency of the ac component & $1 \ldots 20 \mathrm{~Hz}$ \\
Ripple current of the dc component & $1 \mathrm{~A}$ \\
Ripple voltage of the dc component & $1 \mathrm{~V}$ \\
\hline \hline
\end{tabular}

\section{Cooling and Vacuum}

For cooling and baking each coil is provided with a cooling circuit that is equipped with a central heating system. Main parameters of the cooling and baking system are shown in Table 4.

TABLE 4

PARAMETERS OF THE COOLING CIRCUIT

\begin{tabular}{lc}
\hline \multicolumn{1}{c}{ Designation } & Value \\
\hline cooling power & $460 \mathrm{~kW}$ \\
heating power for baking (electric) & $20 \mathrm{~kW}$ \\
max. inlet temperature (baking) & $150{ }^{\circ} \mathrm{C}$ \\
static pressure & $10 \mathrm{bar}$ \\
dynamic pressure & $18 \mathrm{bar}$ \\
mass flow (cooling) & $8.4 \mathrm{~m} / \mathrm{h}$ \\
conductivity & $0.1 \mu \mathrm{SS} / \mathrm{cm}$ \\
\hline \hline
\end{tabular}

The connection between the feed through conductor and the coil winding that is enclosed in the plug-in has to be pluggable and also water tight. To ensure the water tightness and to counter small leakages an inter space vacuum is establishes in this area. The target vacuum is 0.05 mbar whereas the threshold value for pumping is at 0.9 mbar. In case of a leakage a safety valve will open at 2 mbar into a drainage system to avoid a too heavy water load in the vacuum pumps. This threshold will also trigger the alarm for the switch off procedure.

\section{COMMISSIONING}

The commissioning of the $\mathrm{CC}$ system started with operating the coil together with the power supply focusing on the electrical and thermal behavior of the coil and its supply systems. The inter space vacuum was already in operation to observe water leakages. Prior to the start of the W7-X the baking was done for all in-vessel components including the cc.

\section{A. Electrical behavior}

Each coil was operated with 90 different current scenarios, varying the $\mathrm{dc}$ and superimposing the ac component with different amplitudes and frequencies. In the final step all ten coils have been operated with changing dc and ac currents over a time span of about three hours. In figure five the waveforms for current and voltage can be seen for one of the various $\mathrm{dc}+$ ac scenario.

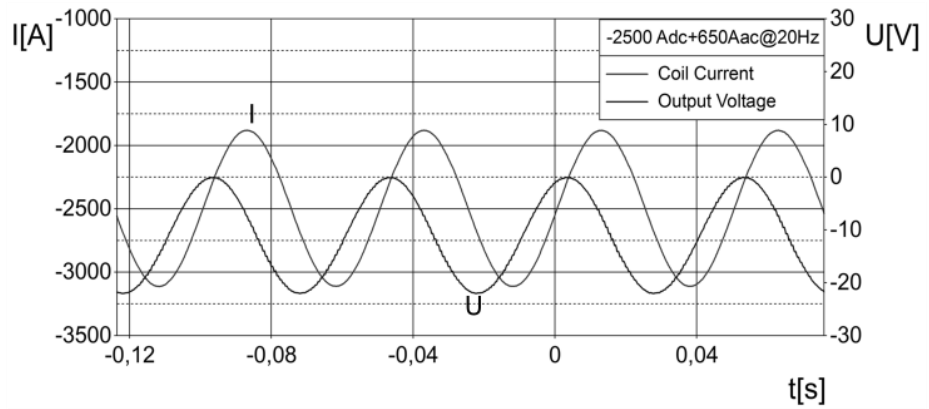

Fig. 5 Current (I) and Voltage (U) for $2500 \mathrm{~A} \mathrm{dc}$ and $625 \mathrm{~A}$ ac at $20 \mathrm{~Hz}$

\section{B. Thermal behavior}

Both CCs and Power supply are water cooled and therefore need special attention with regards to their thermal behavior. The CC and plug-in is not equipped with any sensors whereas the power supply is well monitored and has been already tested during acceptance tests campaigns.

\section{1) Control coil}

There are two critical points in the design of the coil where hot spots can occur, the connection of the plug in to the coil winding and the connection to the current feed. The former is secured by an oversized design of the conductor in the plug-in and the number of lamellas in the plugs to carry the current. The latter is accessible at the outside of the cryostat and was carefully observed during the commissioning. Figure 6 shows the connection area during the heat run after reaching thermal equilibrium. The hot spot can be seen as expected at the clamped block which connects the cable with conductor of the feed through at about $44^{\circ} \mathrm{C}$.

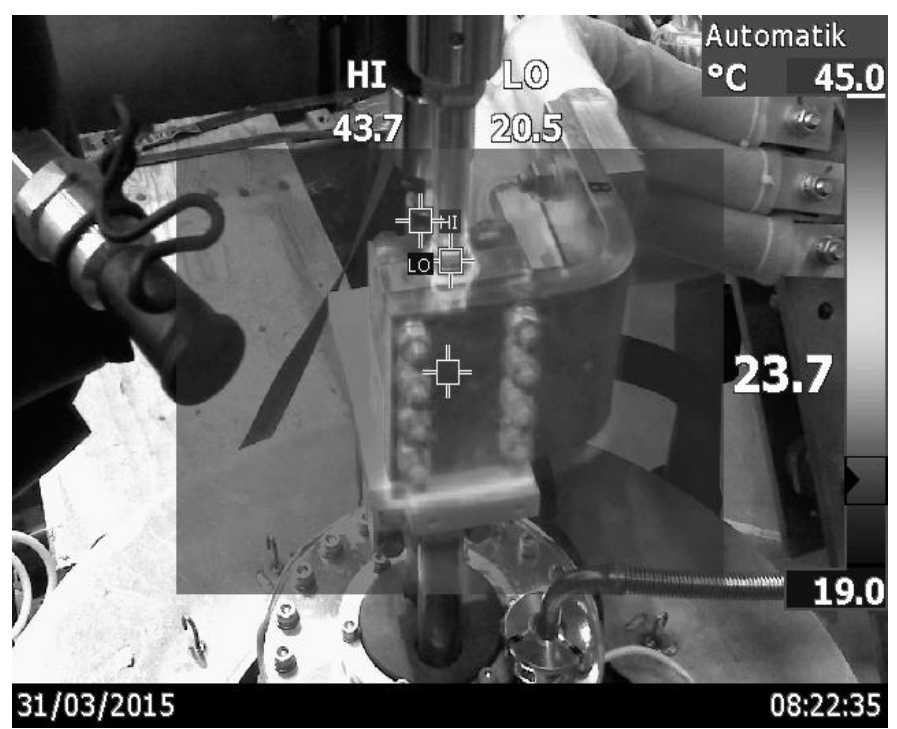

Fig. 6 Thermal picture of the connection area during heat run. The hot spot (HI) with $43.7{ }^{\circ} \mathrm{C}$ is inside the clamped copper block where the contact lamellas are located.

\section{2) Power supply}

The thermal behavior of the power supplies was tested during the test with dummy loads. Focus during these tests was on the water cooled Mosfet inverter which creates the 
highest heat load in the power supply. When operated with the CC the whole power supply plant created significant higher temperatures. The reason behind this is the higher temperature raise in the control coils compared to the dummy loads. This leads to higher ohmic power losses in the coil and therefore to a higher power load for the power supply. Nevertheless the overall temperatures are still below the allowable and not supposed to be critical but as thermal stress leads to aging effects it is desirable to keep the temperatures inside the power supply cabinets as low as possible. Therefore it was decided to install additional venting for the cabinets to compensate the additional heat load.

\section{Inter space vacuum}

After the pumping of the inter space vacuum was started the pumping cycle from target to threshold value was in the range of about three days. During the baking process this cycle got much faster in the range of minutes as expected. After the baking process was finished the cycle was exceeded to about one week except for one coil which still showed constantly a very fast pumping cycle pointing to a leakage. Figure 7 shows how the leakage developed compared to another coil. The investigation revealed a damaged O-ring. Never the less the coil could still be operated. After replacing the damaged Oring this coil is now water tight.

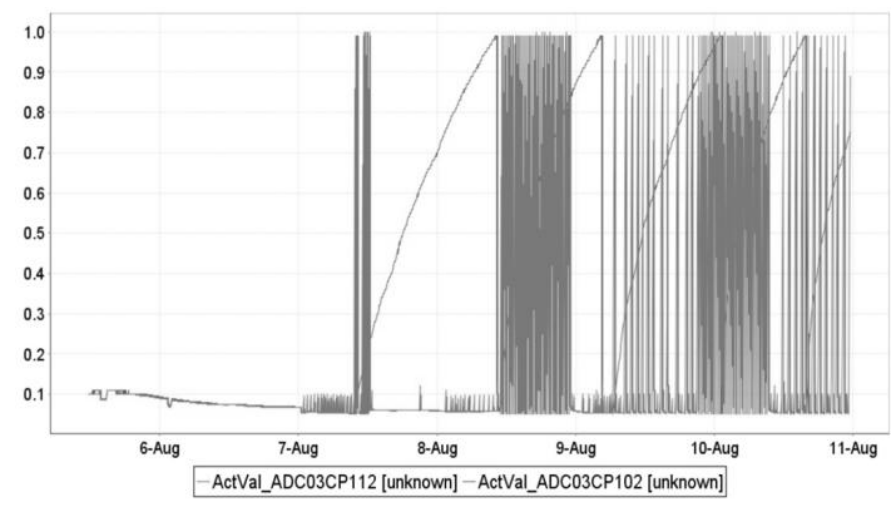

Fig. 7 Pressures in two different coils indicating the leakage in one coil

\section{Baking}

Baking of the in-vessel components is done in four steps. Starting with $50{ }^{\circ} \mathrm{C}$ then increasing to $80{ }^{\circ} \mathrm{C}, 120{ }^{\circ} \mathrm{C}$ and finally $150^{\circ} \mathrm{C}$. The final temperature was never reached as the heater was not able to go beyond $140{ }^{\circ} \mathrm{C}$. The baking was a sufficient success regardless.

The winding pack follows the temperature increases very fast compared to all the other in vessel components. As the coil housing is not thermally attached to the winding pack to allow sliding between them, the housing is mostly heated and cooled by radiation from and to the ambient. This became a problem during cooling down as the winding pack reacted too fast while the ambient and therefore the housing cooled down with a much smaller rate. It was necessary to stop the active cooling of the coil. The passive cool down by conduction through the housing was sufficient to keep the coil winding inside the allowed temperature limits.

\section{LESSONS LEARNED}

\section{A. Zero crossing effect}

Whenever the voltage has to cross zero, while the current remains its polarity during $\mathrm{AC}$ operation, the voltage overshoots which disturbs the current as well. As the controller only calculates effective values for the current and voltage this deviation is not recognized until the current crosses zero again because the controller synchronizes the zero crossing of the current for all ten power supplies. The intensity of this effect is depending on the $\mathrm{du} / \mathrm{dt}$ of the voltage. The lower this value, the higher is the effect. As a consequence, very low frequencies at very small amplitudes will disturb the current beyond the allowed limit for the total harmonic distortion (THD) of $10 \%$. The presence of DC current will negate this effect on the other hand as the needed DC voltage will prevent the overall voltage to cross zeros. Figure 8 shows the effect while the power supply operates with $625 \mathrm{~A}_{\mathrm{P}}$ at $20 \mathrm{~Hz}$.

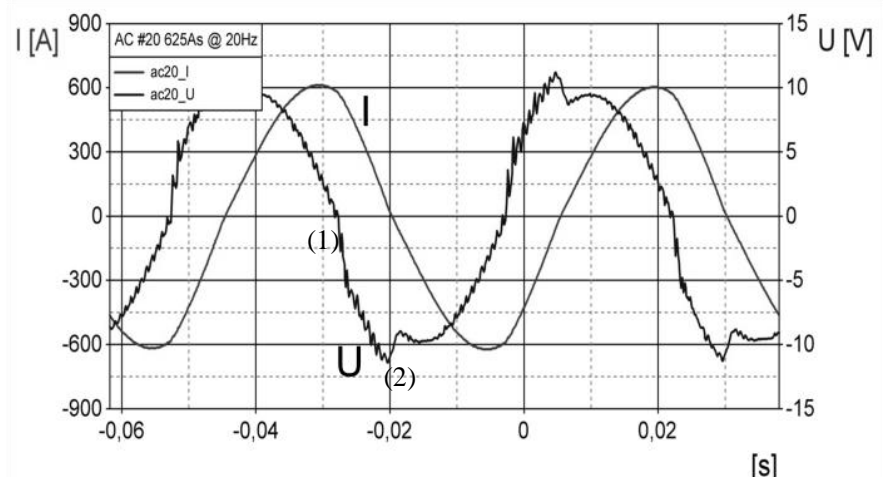

Fig. 8 Zero crossing effect showing how the voltage overshoots (1) when crossing the zero line and gets corrected when the current crosses zero line (2).

An extreme case of the zero crossing effect will happen whenever the dc voltage is more or less equal to the super imposed ac voltage. In that case the zero crossing will occur irregularly at the lowest du/dt possible with the highest effect leading to massive deviations in the peak current. These scenarios need to be avoided by adjusting dc and ac levels accordingly. A redesign of the controller stage to improve the behavior is actually under discussion.

\section{B. Thermal losses during baking}

During the baking process a maximum water inlet temperature of $140^{\circ} \mathrm{C}$ was reached which is below the target temperature of $150^{\circ} \mathrm{C}$. Even that did not hamper the overall baking process it was necessary to investigate the reason. The Insulation of the water lines is sufficient as the losses based on the thermal measurement with about $2.5 \mathrm{~kW}$, is below the calculated $4.4 \mathrm{~kW}$. A check of the installation revealed that the connection area at the feed through is not sufficiently insulated what creates power losses of $8 \mathrm{~kW}$ for all then coils. An added insulation is supposed to prevent these losses in order to reach the projected baking temperature. Also the too fast cooling down of the coil is expected to be reduced by this measure. 


\section{CONCLUSION}

The control coil system of W7-X has been successfully commissioned in 2015. All coils have been operated under full load in steady state operation. Design parameters with regards to cooling, mechanical stresses and electrical operation have been proven sufficient. The zero crossing effect can have an influence on few current scenarios making a redesign of the controller stage necessary. The lack of heating power during the first baking process was counter measured by additional insulation. A leakage in one of the coil connections between coil winding and plug-in was detected but did not prevent the commissioning and was repaired after the first operational phase of W7-X. Furthermore the venting of the PSU cabinets was improved. The CC system is actually ready for operation and to contribute to the next operational phase of W7-X.

\section{REFERENCES}

[1] H.-S. Bosch, V. Bykov, R. Brakel, P. van Eeten, J.-H. Feist, M. Gasparotto, H. Grote, T. Klinger, M. Nagel, D. Naujoks, M. Otte, K. Risse, T. Rummel, J. Schacht, T. Sunn Pedersen, R. Vilbrandt, L. Wegener, A. Werner, R. Wolf, the W-X Team., "Final integration, commissioning and start of the Wendelstein 7-X stellarator operation," Nuclear Fusion (2017), accepted for publication.

[2] [2] R. Stadler. "The in-vessel components of the experiment WENDELSTEIN 7-X" Fusion Engineering and Design 84 (2009) 305-308

[3] [3] Th. Rummel, F. Füllenbach and Th. Mönnich, "Power supplies for the Wendelstein 7-X stellarator", Fusion Engineering and Design, 66-68 (2003), pp. 1115-1118 